(C2006 IEEE. Personal use of this material is permitted. However, permission to reprint/republish this material for advertising or promotional purposes or for creating new collective works for resale or redistribution to servers or lists, or to reuse any copyrighted component of this work in other works must be obtained from the IEEE. 


\title{
A Methodology for Risk Based Decision Making in a Service Oriented Environment
}

\author{
Omar Khadeer Hussain ${ }^{1}$, Elizabeth Chang ${ }^{1}$, Farookh Khadeer Hussain ${ }^{1}$ and Tharam S. Dillon ${ }^{2}$ \\ ${ }^{1}$ School of Information Systems, Curtin University of Technology, Perth, Australia \\ \{Omar.Hussain, Elizabeth.Chang, Farookh.Hussain\}@cbs.curtin.edu.au \\ ${ }^{2}$ Faculty of Information Technology, University of Technology, Sydney, Australia \\ tharam@it.uts.edu.au
}

\begin{abstract}
Decision making is a complex process. It involves in dealing with a lot of uncertainty and projecting in mind what the final outcome might be at the end. Based on the projection of the final outcome a decision is taken. Decision making in a peer-to-peer financial interaction is a further complex process as it also involves with considering the possible financial loss to the trusting agent in the interaction. In this paper we ease the decision making process of a trusting agent to decide whether to interact with a trusted agent or not or choose a particular trusted agent to interact with, by proposing an approach that analyzes the Risk that could be present in interacting with it.
\end{abstract}

\section{Introduction}

The Australian and New Zealand Standard on Risk Management, AS/NZS 4360:2004 states that Risk Identification is the heart of Risk Management [1]. Hence, Risk should be identified in order to analyze and manage it better. Risk analysis is the science of evaluating Risks resulting from past, current, anticipated or future activities. The use of these evaluations includes providing information for determining regulatory actions to limit Risk and for decision making. Risk analysis is an interdisciplinary science that relies on laboratory studies, collection and exposure of data and computer modelling. Risk is seen as a combination of:

a) The uncertainty of the outcome; and

b) The cost of the outcome when it occurs, usually the loss incurred, which is related to Risk.

For the trusting agent to decide whether to interact or not with a trusted agent or to choose an agent to interact with from a set of possible trusted agents, it should first analyze the Risk that could be involved in dealing with the particular trusted agent. Risk in a service oriented environment is defined the likelihood that the trusted agent might not act as expected according to the trusting agent's expectations in a given context and at a particular time once the interaction begins, resulting in the loss of $\$$ and the resources involved in the interaction [2].

In order to analyze the Risk in an interaction we defined the term Riskiness and the Riskiness scale. A Riskiness value represents a level of Risk on the Riskiness scale. The Riskiness scale as shown in figure 1 is in the domain of $(-1,5)$. Each value within this domain on the Riskiness scale represents a level of Risk. The Riskiness value is assigned to the trusted agent by the trusting agent after assessing the level of un-commitment in its actual behavior with respect to the promised behaviour or promised commitment. The process of assigning a Riskiness value to the trusted agent is defined in Hussain et al [2].The Riskiness value that the trusted agent gets from the trusting agent is dependent on a number of accessing criteria in their interaction. The accessing criteria are defined as the set of factors or bases against which the un-committed behavior of the trusted agent is going to be determined or assessed. The accessing criteria are derived from the expected behavior or the mutually agreed behavior. We term the accessing criteria as the criteria. So the criteria for determining the Riskiness of a trusted agent are not same for all the interactions. They vary according to each trusting agent. Hence even in a same context the criteria of two trusting agents for assessing the un-committed behavior of a particular trusted agent to assign it a Riskiness value might be different from each other.

Risk analysis by the trusting agent to decide whether to interact or not with the trusted agent can be done by:

1. Analyzing the previous Riskiness values of the possible trusted agent according to the specific criteria of the trusting agent's future interaction with it.

2. Determining the future Riskiness value of the possible trusted agent till the time slot of the trusting agent's interaction with it.

3. Determining the possible loss to the trusting agent's resources if it interacts with a possible trusted agent

In this paper we will highlight the above mentioned factors that can be utilized by the trusting agent to analyze the possible Risk in its interaction with a possible trusted agent. This analysis will help the trusting agent to decide whether to interact or not with 


\begin{tabular}{|c|c|c|c|}
\hline $\begin{array}{l}\text { Riskiness } \\
\text { Levels }\end{array}$ & $\begin{array}{c}\text { Magnitude of } \\
\text { Risk } \\
\end{array}$ & $\begin{array}{c}\text { Riskiness } \\
\text { Value }\end{array}$ & Star Rating \\
\hline Unknown Risk & & -1 & Not Displayed \\
\hline Totally Risky & $\begin{array}{l}91-100 \% \text { of } \\
\text { Risk }\end{array}$ & 0 & Not Displayed \\
\hline $\begin{array}{l}\text { Extremely } \\
\text { Risky }\end{array}$ & $\begin{array}{l}71-90 \% \text { of } \\
\text { Risk }\end{array}$ & 1 & 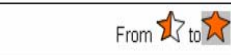 \\
\hline Largely Risky & $\begin{array}{l}51-70 \% \text { of } \\
\text { Risk }\end{array}$ & 2 & From $\Sigma$ \\
\hline Risky & $\begin{array}{l}26-50 \% \text { of } \\
\text { Risk }\end{array}$ & 3 & From $\sum$ \\
\hline $\begin{array}{l}\text { Largely } \\
\text { UnRisky }\end{array}$ & $\begin{array}{l}11-25 \% \text { of } \\
\text { Risk }\end{array}$ & 4 & From \\
\hline UnRisky & $\begin{array}{l}0-10 \% \text { of } \\
\text { Risk }\end{array}$ & 5 & From \\
\hline
\end{tabular}

Figure 1. Showing the Riskiness scale and its associated levels

the particular trusted agent or to choose an agent to interact with among a set of possible trusted agents.

\section{Determining the previous Riskiness value of the Trusted Agent}

If the trusting agent has a previous interaction history with the trusted agent in the same context and in the recent time slot as its future interaction, then it can decide whether to interact or not with that particular trusted agent based on the Riskiness value that it assigned in their previous interaction. On the contrary if the trusting agent has a previous interaction history with the trusted agent in a different context and time as compared to its future interaction or does not have any previous interaction history at all with the trusted agent, then it can decide whether to interact or not with the trusted agent by analyzing its Riskiness value after soliciting for its recommendations from other agents.

\subsection{Soliciting for Recommendations}

The trusting agent will solicit for recommendations about the particular trusted agent from the agents present on the network by specifying the context of its future interaction and its criteria. The agents who reply back with the recommendations are called as the Recommending agents. A recommendation can be given by any agent present on the network. However, it is highly unlikely that the recommendations communicated by the recommending agents would be completely reliable. It is possible that some recommending agents might be communicating untrustworthy recommendations. The recommendations can be classified into three categories namely trustworthy, untrustworthy and unknown recommendations. The trusting agent assimilates the recommendations from the trustworthy and unknown recommending agents and ignores those from the untrustworthy recommending agents, as the Risk in considering those recommendations might be high. The process of classifying the recommendations as trustworthy, unknown and untrustworthy is discussed in Hussain et al [3]. For just understanding the concept let us consider that a recommending agent having a Riskiness value while giving recommendation (RRP) in the range of $(-1,1)$ is said as a trustworthy recommending peer.

The recommending agents reply back with the Risk Set as their recommendation. The Risk set contains the recommended Riskiness value for the particular trusted agent, as recommended by the recommending agent, depending on their last interaction with it. As explained in Hussain et al [4] the Risk Set is an ordered way for the recommending agent to represent the various details of its last interaction with the particular trusted agent, so that the trusting agent asking for recommendations can know the meaning of each element in the recommendation and consider only those recommendations in determining the Riskiness value of the trusted agent whose criteria are of interest to it in its future interaction. The format of the Risk set is:

\{TP1, TP2, Context, CR, R', (Criteria, Commitment level), R, Cost, Start time, End time, RRP

Where: TP1 was the trusting agent in the interaction. It is also the recommending agent while giving recommendations,

TP2 was the trusted agent in the interaction,

Context represents the context of the interaction,

CR represents the Current Riskiness value of the trusted agent before the interaction, which is achieved either by the last interaction of the trusting/recommending agent with the trusted agent in the same time slot or by asking recommendations from other agents and assimilating them to determine the Riskiness value of the trusted agent according to the criteria of the trusting agent in the interaction,

R' shows the predicted Riskiness value of the trusted agent depending on its past values,

(Criteria, Commitment level) shows the factors or bases which the recommending agent used in its interaction with the trusted agent to assign it a Riskiness value. These criteria are necessary to mention while giving recommendations, so that a trusting agent who asks for recommendation knows the factors or bases on which this particular trusted agent was assigned the recommended Riskiness value and can take only those recommendations which are of 
interest to it according to the criteria of its future interaction. Commitment level specifies whether the particular criterion was fulfilled by the trusted agent or not. A value of either 0 or 1 is assigned here based on the evaluation of the particular criterion measuring for its fulfillment according to expected behavior. Further explanation is given in Hussain et al [2],

$\mathrm{R}$ is the Riskiness value assigned by the recommending agent to the trusted agent after the interaction. This is the Riskiness value being recommended for the trusted agent,

Cost represents the cost of the interaction,

Start Time is the time at which the recommending agent started the interaction with the trusted agent,

End time is the time at which the interaction of the recommending agent ended with the trusted agent,

RRP is the Riskiness value of the recommending agent while giving recommendations. This value is used to determine whether the recommendation is trustworthy or not.

\subsection{Assimilating the Recommendations}

The recommendations that the trusting agent receives for the particular trusted agent will be according to the recommending agent's criteria in its interaction. The criteria of the trusting agent in its future interaction with the trusted agent might be different as compared to those of the recommending agents. In this case the Riskiness values that the recommending agents recommend to the trusting agent might not be of any use to it if their criteria don't match. But the trusting agent can assimilate the recommendations according to the criteria in its future interaction with the trusted agent to determine the trusted agent's Riskiness value.

The Riskiness value of a particular trusted agent ' $\mathrm{P}$ ' in criterion ' $\mathrm{C}$ ' $\left(\mathrm{R}_{\mathrm{PC}}\right)$ can be determined after assimilating the recommendations by using the following formulae:

$$
\begin{gathered}
\left(\alpha * \left(\left(\frac{1}{N} * \mid \gamma *\left(\sum_{i=1}^{N} \operatorname{RRP}_{\mathrm{i}} * \text { Commitment Level }_{\mathrm{i}}\right) \mid\right)+\right.\right. \\
\left.\left.\left(\frac{1}{K} * \mid \delta *\left(\sum_{l=1}^{K} \operatorname{RRP}_{1} * \text { Commitment Level }_{1}\right) \mid\right)\right)\right) \\
+{ }^{\prime} *\left(\left(\frac{1}{J} * \gamma\left(\sum_{o=1}^{J} \text { Commitment Level } \mathrm{o}\right)\right)+\right. \\
\left.\left.\quad\left(\frac{1}{M} * \delta\left(\sum_{q=1}^{M} \text { Commitment Level }_{\mathrm{q}}\right)\right)\right)\right)
\end{gathered}
$$$$
\text { Equation ---1 }
$$

Where: $\mathrm{RRP}_{\mathrm{i}}$ is the Riskiness value of the trustworthy recommending agent $\mathrm{i}$, whose recommendation is in the recent time slot of the trusting agent's interaction,

$\mathrm{RRP}_{1}$ is the Riskiness value of the trustworthy recommending agent 1 , whose recommendation is in the far recent time slot,

Commitment level is the level of commitment by the trusted agent in the particular criterion ' $c$ ' as recommended by the recommending agents in their recommendations,

$\mathrm{N}$ and $\mathrm{K}$ are the number of trustworthy recommendations classified according to the time slot of the recommendations,

$\mathrm{J}$ and $\mathrm{M}$ are the number of unknown recommendations classified according to the time slot of the recommendations,

$\gamma$ and $\delta$ are the weights attached to the recent time slot recommendations and far recent time slot recommendations respectively. These weights give more significance to recommendations which are in the recent time slot as compared to the far recent ones. In general $\gamma>\delta$ and $\gamma+\delta=1$,

$\alpha$ and $\beta$ are the weights attached to trustworthy recommendations and unknown recommendations respectively. These weights give more significance to recommendations from the trustworthy recommending agents as compared to the ones from unknown recommending agents. In general $\alpha>\beta$, and $\alpha+\beta=1$.

The first part of the above equation calculates the Riskiness value of the trusted agent ' $\mathrm{P}$ ' in a criterion ' $\mathrm{C}$ ' by taking the recommendations of the trustworthy recommending agents and the second part calculates the Riskiness value of the same trusted agent in the same criterion ' $C$ ' by taking the recommendations of the unknown recommending agents. The recommendations from the untrustworthy recommending agents are left out and not considered. Further the Riskiness value determination of the trusted agent by taking the recommendations of the trustworthy and the unknown recommending agents too is done in two parts according to the time slot of the recommendations. The trusting agent should give more weight to the recommendations which are in the recent time slot of its interaction as compared to the far recent time slot recommendations. Those weights are represented by $\gamma$ and $\delta$ respectively. In order to give more importance to the recommendations from the trustworthy recommending agents as compared to the recommendations from the unknown recommending agents, weights are attached to the two parts of the equation. These weights are represented by $\alpha$ and $\beta$ respectively. It depends upon the trusting agent on how much weight does it want to give to each recommendation. By multiplying the Riskiness value of the recommending agent (RRP) with the 
commitment level that it is suggesting for a criterion we are getting the accurate recommendation according to its Riskiness.

As mentioned earlier any recommending agent whose Riskiness value while giving recommendations is with in the range of $(-1,1)$ is said to be a trustworthy recommending agent. So it is possible that the Riskiness value for the trusted agent in a criterion ' $\mathrm{C}$ ' calculated from the trustworthy recommendations might come negative. We take the range of $(-1,1)$ to determine whether the recommendation from the recommending agent is trustworthy or not and once it has been determined, it should not have any effect in determining the final Riskiness value of the trusted agent in a criterion by assimilating the recommendations. Hence we apply the mod operator to the first part of equation 1 which determines the Riskiness of the trusted agent in a criterion ' $\mathrm{C}$ ' by taking the trustworthy recommendations.

In order to map the Riskiness value $\left(\mathrm{R}_{\mathrm{PC}}\right)$ of the trusted agent ' $\mathrm{P}$ ' in a criterion ' $\mathrm{C}$ ' on the Riskiness scale (RS), it should be multiplied by 5 . Hence Riskiness value of the trusted agent ' $\mathrm{P}$ ' in a criterion ' $\mathrm{C}$ ', mapped to the Riskiness scale ( $\left.\mathrm{R}_{\mathrm{PRSC}}\right)$ is:

$$
\mathrm{R}_{\mathrm{PRSC}}=\mathrm{ROUND}\left(\mathrm{R}_{\mathrm{PC}} * 5\right) \quad \text { Equation ---2 }
$$

When the Riskiness value in each criterion of the trusting agent's interaction has been determined on the Riskiness scale for the trusted agent by assimilating the recommendations, then the final Riskiness value of the trusted agent in the interaction can be determined by weighing the individual Riskiness value of each criterion according to its significance, depending on the trusting agent. The levels of significance for each criterion $(\mathrm{Sc})$ are shown in table 1 . The significance of each criterion in an interaction might depend on the degree to which it influences the successful outcome of the interaction according to the trusting agent.

\begin{tabular}{|c|c|}
\hline $\begin{array}{l}\text { Significance level of } \\
\text { the Criterion (Sc) }\end{array}$ & $\begin{array}{l}\text { Significance Rating and } \\
\text { Semantics of the level }\end{array}$ \\
\hline 1 & Minorly Significant \\
\hline 2 & Moderately Significant \\
\hline 3 & Largely Significant \\
\hline 4 & Majorly Significant \\
\hline 5 & Highly or Extremely Signifcant \\
\hline
\end{tabular}

The final Riskiness value $\left(\mathrm{CR}_{\mathrm{P}}\right)$ of the trusted agent ' $\mathrm{P}$ ' according to the criteria and significance of each criterion in the interaction by soliciting recommendations from other agents can be calculated as:

$$
\mathrm{CR}_{\mathrm{p}}=\operatorname{ROUND}\left(\frac{1}{\sum_{c=1}^{n} S_{c}}\left(\sum_{c=1}^{n} \mathrm{Sc}^{*} \mathrm{R}_{\mathrm{PRSC}}\right)\right)
$$

Equation --- 3

Where $\mathrm{Sc}$ is the significance of the criterion ' $\mathrm{C}$ '

$\mathrm{R}_{\text {PRSC }}$ represents the Riskiness value of the trusted agent ' $\mathrm{P}$ ' in criterion ' $\mathrm{C}$ ' on the Riskiness scale

$\mathrm{n}$ is the number of criterions in the interaction.

It should be noted that the Riskiness value of the trusted agent $\left(\mathrm{CR}_{\mathrm{p}}\right)$ determined by assimilating the recommendations should be set to 0 if it is less than 0 , as the Riskiness scale ranges from 0 to 5 with a value of -1 as Unknown Risk.

\section{Determining the Future Riskiness Value of the Trusted Agent}

The trusting agent can analyze the Risk in the interaction better and decide whether to interact or not with a trusted agent, if it predicts the Riskiness value of that trusted agent over the time period of its interaction with it. We define the total boundary of time which the trusting agent takes into consideration to analyze and predict the Riskiness value of the trusted agent as the time space. Risk is dynamic and it varies from time to time. The total time of interaction between the trusting agent and trusted agent depends on the context. If the time space is very big, then it is not possible for the trusting agent to have the same impression for the trusted agent throughout that it had at a particular time. Hence the total time space is divided into different non-overlapping parts and the Riskiness value of the trusted agent is analyzed and predicted in each of those parts. These different nonoverlapping parts are called as time slots.

The trusting agent in order to predict the reputation or the Riskiness values of a trusted agent at time ' $t+1$ ' to analyze the Risk in the interaction, should know its reputation or Riskiness value till time ' $t$ '. If the trusting agent hasn't previously interacted with the trusted agent in the context of its present interaction, then it can determine its previous reputation or Riskiness values till time ' $t$ ' by soliciting recommendations from other agents as described in the previous section. We propose that the trusting agent in order to predict the future Riskiness value of a trusted agent at time ' $t+1$ ' in a particular context, should consider all the trustworthy and unknown recommendations from the recommending agents for that particular trusted peer in that particular context to determine the Riskiness value of the trusted agent till time' $t$ '. 
In order to predict the future Riskiness value of the trusted agent, the trusting agent has to first decide about the total time space over which it is going to analyze the behavior and the Riskiness values of the trusted agent. Within the time space, the trusting agent should determine the duration of each time slot in which it will analyze the behavior of the trusted agent. Once it knows the duration of each slot it can determine the number of time slots in the given time space and analyze its reputation or Riskiness value in each time slot.

Our method of Riskiness value prediction for the trusted agent at time ' $t+1$ ' is by accumulating the Riskiness values of the trusted agent till time ' $t$ ' and utilize the Gaussian Distribution to determine the probability of the future Riskiness value within each range of the Riskiness scale. The rounded Riskiness value corresponding to the range of the highest probability is the future Riskiness value of the trusted agent at time ' $t+1$ '. As mentioned in section 1, the Riskiness scale ranges from $(-1,5)$, with -1 denoted as Unknown Risk. So the future Riskiness value of a trusted agent is predicted in the domain of $(0,5)$.

To determine the probability of the trusted agent's future Riskiness value within a range on the Riskiness scale, let us suppose that the trusting agent has collected recommendations from other agents according to the context and criteria of its interaction. Those recommendations are represented as:

$$
\left\{R_{1}, R_{2}, R_{3} \ldots R_{K}\right\}
$$

where $\mathrm{K}$ is the number of recommendations.

The Mean Riskiness Value $(\mu)$ is calculated as:

$$
\mu=\frac{1}{K} \sum_{i=1}^{K} R_{\mathrm{i}} \quad \text { Equation --- } 4
$$

Accordingly the unbiased Sample Variance $\left(\sigma^{2}\right)$ is:

$$
\sigma^{2}=\frac{1}{K-1} \sum_{i=1}^{K}\left(R_{i}-\mu\right)^{2} \quad \text { Equation --- 5 }
$$

The future Riskiness value of a trusted agent is denoted by R'. Since R' $\sim\left(\mu, \sigma^{2}\right)$, then for any random variable $\mathrm{R}^{\prime}$ according to Gaussian distribution, the probability of $\mathrm{R}^{\prime}$ in a given range can be expressed as [5]:

$$
\mathrm{P}\left(\mathrm{a}<\mathrm{R}^{\prime}<\mathrm{b}\right)=\frac{1}{\sqrt{2 \prod} \sigma} \int_{\frac{a-\mu}{\sigma}}^{\frac{b-\mu}{\sigma}} \mathrm{e}^{\frac{-t 2}{2}} \mathrm{dt}
$$

$$
\text { Equation --- } 6
$$

Alternatively for any random variable R', its Cumulative Distributive Function (CDF) denoted by $\Phi$ in the range $-\infty<\Gamma<\infty$ is expressed in terms of its probability density function as [6]:

$$
\Phi(г)=\int_{-\infty}^{r} \frac{1}{\sqrt{2 \prod}} \mathrm{e}^{\frac{-t 2}{2}} \mathrm{dt}
$$

Similarly the probability of $\mathrm{R}^{\prime}$ in a given range, in terms of $\Phi$, the standard normal cumulative distributive function is determined by [6]:

$$
\begin{aligned}
& \mathrm{P}\left(\mathrm{a}<\mathrm{R}^{\prime}<\mathrm{b}\right)=\Phi\left(\frac{b-\mu}{\sigma}\right)-\Phi\left(\frac{a-\mu}{\sigma}\right) \\
& \text { Equation --- } 7
\end{aligned}
$$

As mentioned earlier the probability of the future Riskiness value R' within the domain of $(0,5)$ on the Riskiness scale is determined. The rounded Riskiness value corresponding to the range which has the highest probability based on the past Riskiness values is chosen as the future Riskiness value of the trusted agent.

\section{Quantifying Risk in Financial terms}

\subsection{Factors for Determining Financial Loss}

The financial loss to a trusting agent in an interaction is dependent on:

1. Its previous interaction history with the trusted agent, if any, or on the reputation of the trusted agent determined by soliciting recommendations from other agents according to the criteria of the trusting agent's interaction;

2. Predicting the future Riskiness value of the trusted agent in the time space of the trusting agent's interaction;

3. Willingness of the trusting agent in interacting with the trusted agent;

4. Familiarity of the trusting agent with the medium of interaction;

5. Familiarity of the trusting agent with the trusted agent; and

6. Nature of the trusting agent.

In the next sub-section, we will explain these factors in detail and define the metrics which are used to quantify each of these factors and express them numerically. These factors will be considered when quantifying Risk in financial terms.

\subsubsection{Previous interactions or considering reputation from other agents}

The outcome of previous interactions, if any, between the trusting agent and the trusted agent will 
help in analyzing the Risk that could be present in future interactions between them. Depending on the outcome of the previous interaction, the trusting agent might be able to make a decision to trust the trusted agent or not. If the outcome of the previous interaction was positive and it concluded according to expected behaviour, then the trusting agent might have some belief in the trusted agent and may proceed with any future interactions. Consequently, the Risk associated with this interaction might not be high, as there is some belief present among them. On the contrary, if the outcome of the previous interaction was negative then the trusting agent may have some doubts about proceeding with any future interaction with the trusted agent and fears the Risk involved in the interaction may be too high.

If there is no previous interaction history between the trusting and the trusted agents, then the trusting agent can analyze the Risk that could be present in its interaction with the trusted agent by enquiring about its reputation in the particular context of this interaction. As discussed in section 2, reputation of a trusted agent can be determined by soliciting for recommendations from other agents and later assimilating the information.

We represent the Riskiness value of the trusted agent before starting an interaction, that is achieved either by the previous interaction history of the trusting agent with the trusted agent or by soliciting for its recommendations from other agents by the metric Pre Trusted Agent. The value of the metric Pre Trusted Agent is between $(0,5)$ on the Riskiness scale.

\subsubsection{Predicting the future Riskiness value of the trusted agent}

Risk varies according to time. It is not possible for a trusting agent to have the same impression of the trusted agent that it had at a particular time throughout all interactions. The trusting agent, in order to determine the possible financial loss in an interaction accurately should take into consideration the future Riskiness value of the trusted agent in the time space of their interaction. The future Riskiness value for the trusted agent is predicted based on recommendations acquired from the recommending agents according to the context and criteria of the trusting agent's interaction. The process of classifying the time of the trusting agent's interaction with the trusted agent into different timeslots and predicting the future Riskiness value of the trusted agent in each timeslot is defined in section 3.

We represent the future Riskiness value of the trusted agent within the given time space of the trusting agent's interaction by the metric Fut Trusted Agent. The predicted future Riskiness value of the trusted agent Fut Trusted Agent will be in the range of $(0,5)$ on the Riskiness scale.

\subsubsection{Willingness of the trusting agent to interact with the trusted agent}

The financial loss in an interaction also depends on the commitment / willingness of the trusting agent in dealing with the trusted agent. It depends on the degree of how comfortable the trusting agent is in dealing with the trusted agent. The trusting agent will be more comfortable in dealing with the trusted agent if it has a favourable previous interaction history and there was a high degree of parallelism between the expected and actual behaviours. The degree of parallelism between the expected behaviour and the actual behaviour is represented by the Riskiness value that is assigned after the interaction. The greater degree of parallelism between these two behaviours, Expected and Actual, indicates that the trusted agent has acted according to the expected behaviour and the Risk involved in the interaction was less. Hence, the trusting agent might be content in interacting with the trusted agent and might proceed with another interaction with it in the near future as they have established a significant level of trust between them.

We define the willingness of the trusting agent to interact with the trusted agent by the metric Will Interaction. In order to measure the willingness of the trusting agent in interacting with the trusted agent we define 3 levels of the metric Will Trusting Agent. Those levels are defined in Table 2.

\begin{tabular}{|c|l|}
\hline $\begin{array}{l}\text { Level of the Metric } \\
\text { Will Trusting Agent }\end{array}$ & \multicolumn{1}{c|}{ Semantics of the level } \\
\hline 0 & $\begin{array}{l}\text { The trusting agent is not at all willing to } \\
\text { deal with the trusted agent }\end{array}$ \\
\hline 1 & $\begin{array}{l}\text { The trusting agent is comfortable in } \\
\text { dealing with the trusted agent but would } \\
\text { prefer in dealing with other agents if } \\
\text { possible }\end{array}$ \\
\hline 2 & $\begin{array}{l}\text { The trusting agent is totally comfortable } \\
\text { in dealing with the trusted agent }\end{array}$ \\
\hline Table 2 showing the levels for the metric Will Interaction
\end{tabular}

\subsubsection{Familiarity of the trusting agent with the medium of interaction}

The possible degree of financial loss in the trusting agent's resources also varies according to the familiarity of the trusting agent towards the medium of interaction with the trusted agent. If the trusting agent is familiar with the medium in which it is going to interact then it will ease the Risk that could be present. We represent the familiarity of the trusting agent with the medium of interaction by the metric Fam Medium. In 
order to measure the familiarity of the trusting agent with the medium of interaction we define two levels for the metric Fam Medium. Those levels are defined in Table 3.

\begin{tabular}{|c|l|}
\hline $\begin{array}{c}\text { Level of the } \\
\text { Metric Fam Medium }\end{array}$ & \multicolumn{1}{c|}{ Semantics of the level } \\
\hline 0 & $\begin{array}{l}\text { The trusting agent is not familiar with the } \\
\text { medium of the interaction }\end{array}$ \\
\hline 1 & $\begin{array}{l}\text { The trusting agent has previous experience } \\
\text { and is familiar with the medium of the } \\
\text { interaction }\end{array}$ \\
\hline
\end{tabular}

Table 3 showing the level for the metric Fam Medium

\subsubsection{Familiarity of the trusting agent with the trusted agent}

If the trusting agent and the trusted agent are familiar with each other as a result of previous interactions, then the Risk that could be present in their future interactions can be analyzed easily depending on the Riskiness values that were assigned in their previous interactions. In order to determine the potential financial loss in an interaction between the trusting agent and the trusted agent it is important to consider if the trusting agent is familiar with the trusted agent or not. The familiarity of the trusting agent with the trusted agent is represented by the metric Fam Trusted Agent. In order to measure the familiarity of the trusting agent with the trusted agent we define two levels of the metric Fam Trusted Agent as shown in Table 4.

\begin{tabular}{|c|l|}
\hline $\begin{array}{l}\text { Level of the } \\
\text { metric Fam Trusted } \\
\text { Agent }\end{array}$ & \multicolumn{1}{c|}{ Semantics of the level } \\
\hline 0 & $\begin{array}{l}\text { The trusting agent has not interacted with } \\
\text { the trusted agent before and is not at all } \\
\text { familiar with it }\end{array}$ \\
\hline 1 & $\begin{array}{l}\text { The trusting agent has previous interaction } \\
\text { history with the trusted agent and is familiar } \\
\text { with it. }\end{array}$ \\
\hline
\end{tabular}

Table 4 showing the level for the metric Fam Trusted Agent

\subsubsection{Nature of the trusting agent}

Another factor which varies Risk in an interaction is the nature and thinking attitude of the trusting agent, which decides on how it will act and react in certain situations. The nature of the trusting agent plays an important role in deciding whether to proceed with the interaction or not, thus help in determining the financial loss that could be involved in an interaction.

If the trusting agent has an impatient nature or acts in haste, then there is a possibility that he might proceed with the interaction without looking at the previous history of the trusted agent or soliciting for recommendations, hoping to achieve the desired outcome as soon as possible. The Risk associated in these interactions might be high. The trusting agent might care for personal values and start an interaction. For example let us suppose that the trusting agent and the trusted agent might have dealt successfully before in a different context and in a different interaction. Now, they are dealing again in a context and in an interaction in which the trusted agent does not have any experience. Yet the trusting agent might care for personal values and might be ready to take the extra Risk involved and proceed with the interaction. On the contrary, the trusting agent might have a cautious nature of proceeding according to the results and feedback of the previous interactions of the trusted agent. In this case, it might not be willing to trust the trusted agent in any other context in which it does not have any experience. He might feel the Risk involved in such an interaction could be high and might not proceed in interacting with him. Hence, the Risk too decreases according to the trusting agent's decision. If the trusting agent decides to go ahead in an interaction according to the correct way, i.e. in a logical fashion not caring about the personal values then it will be making the decision to proceed with the interaction only if the expected advantages outweigh the negative factors. The Risk involved in such interactions might be less when compared to the other ones.

The trusting agent might decide to proceed in the interaction or not, depending on its nature and it is important to take that into consideration while determining the financial loss in an interaction. The nature of the trusting agent is represented by the metric Nat Trusting Agent. We define 3 levels of the metric Nat Trusting Agent in order to determine the nature of the trusting agent. Those levels are defined in Table 5.

\begin{tabular}{|c|l|}
\hline $\begin{array}{l}\text { Level of the } \\
\text { metric Nat Trusting }\end{array}$ & \multicolumn{1}{c|}{ Semantics of the Level } \\
\hline Agent & $\begin{array}{l}\text { The trusting agent is impulsive in nature and } \\
\text { acts in haste. It prefers proceeding in an } \\
\text { interaction even if there is high Risk or } \\
\text { without analyzing the Risk that could be } \\
\text { present in an interaction. }\end{array}$ \\
\hline 1 & $\begin{array}{l}\text { The trusting agent is conservative in nature } \\
\text { and prefers taking some Risk. It might also } \\
\text { care for personal values. }\end{array}$ \\
\hline 2 & $\begin{array}{l}\text { The trusting agent is determined in nature and } \\
\text { will proceed in the interaction only if the } \\
\text { expected advantages outweigh the negative } \\
\text { factors. }\end{array}$ \\
\hline
\end{tabular}

Table 5 showing the level for the metric Nat Trusting Agent

\subsection{Determining the Possible Financial Loss in an Interaction}

In order to determine the possible financial loss in an interaction the trusting agent, by making use of the above mentioned metrics, should derive a numerical 
value which quantifies its possible interaction with the trusted agent. The trusting peer's possible interaction can be quantified by assigning a level for each metric according to the possible future state of the interaction and later adding the individual value of each metric. We represent the numerical value quantifying the possible interaction of the trusting agent with the trusted agent as Poss Interaction.

$$
\begin{aligned}
& \text { Hence Poss } \text { Interaction }= \\
& \text { Pre }{ }_{\text {Trusted Agent }}+\text { Fut } \text { Trusted Agent }+ \text { Will } \\
& \text { Trusting Agent } \\
& \text { Medium }+ \text { Fam } \text { Trusted Agent }^{+ \text {Nat }}{ }_{\text {Trusting Agent }}
\end{aligned}
$$

\section{Equation --- 8}

Once the numerical value quantifying the possible interaction between the trusting agent and the trusted agent has been determined, it should be compared with the numerical value which quantifies an interaction between the same agents with no financial loss at all to the trusting agent's resources. This value is achieved by the above defined metrics by substituting the individual values for each metric in Poss Interaction that would represent a totally non-risky interaction. We represent the numerical value which quantifies an interaction with no financial loss at all to the trusting agent's resources as Noloss Interaction.

$$
\begin{aligned}
& \text { Hence Noloss }{ }_{\text {Interaction }}= \\
& \text { Pre }{ }_{\text {Trusted Agent }}+\text { Fut Trusted Agent }+ \text { Will }{ }_{\text {Trusting Agent }}+\text { Fam } \\
& \text { Medium }+ \text { Fam Trusted Agent }+ \text { Nat Trusting Agent }
\end{aligned}
$$$$
\text { Equation --- } 9
$$

We define Loss Interaction as the metric which expresses in numerical value the possible level of loss that could be in an interaction. This is achieved by comparing the difference of the numerical values representing a totally non-risky interaction and the possible way in which the interaction might proceed with respect to the numerical value that expresses an interaction with no financial loss at all. The metric Loss Interaction depicts the level of financial loss that could be possible in an interaction to the trusting agent with the trusted agent. Determining Loss ${ }_{\text {Interaction by: }}$

$$
\begin{array}{r}
\text { LosS }_{\text {Interaction }}=\frac{\text { NolosSInteraction }}{\text { NolosSInteraction }} \\
\text { Equation }--\mathbf{1 0}
\end{array}
$$

(Cost of the Interaction * Loss Interaction

$$
\text { Equation --- } 11
$$

The possible percent of loss in an interaction can be determined as:

Possible Percent of Loss Interaction $=\left(\operatorname{Loss}_{\text {Interaction }} * 100\right)$

$$
\text { Equation --- } 12
$$

\section{Conclusion}

In this paper we defined the methodology that will assist the trusting agent to decide or conclude whether to interact or not with a particular trusted agent. This approach can also be utilized by the trusting agent to choose a particular agent to interact with among a set of possible trusted agents. This approach addresses some important factors related to Risk. The trusting agent can reach at a conclusion by analyzing the possible Risk that could be present in the interaction by considering these factors.

\section{References}

[1] D.F. Cooper, "The Australian and New Zealand Standard on Risk Management, AS/NZS 4360:2004", Tutorial Notes: Broadleaf Capital International Pty Ltd., 2004 Available:http://www.broadleaf.com.au/tutorials/Tut_Standar d.pdf

[2] O.K. Hussain, E. Chang, F.K.Hussain and T.S. Dillon, “A Methodology for Risk Measurements in e-Transactions", Special issue of International Journal of Computer System, Science and Engineering, CRL publishing Ltd, UK., To be published in March/April 2006.

[3] O.K Hussain, E.Chang, F.K. Hussain, T.S. Dillon and B. Soh, "Context Based Riskiness Assessment", IEEE TENCON 2005, pp. 352-356, Melbourne, November 22-24 2005.

[4] O.K Hussain, E.Chang, F.K. Hussain, T.S. Dillon and B. Soh, "Modeling the Risk Relationships and Defining the Risk Set" Proceedings of CollECTeR Latam 2005, Chile, 3-5 October 2005, pp 1-9.

[5] N.A. Weiss, 'A Course in Probability', Pearson Education Inc. 2005, ISBN 0-201-77471-2

[6] A.F. Karr, 'Probability', Springer-Verlag New York, Inc. 1993, ISBN 0-387-94071-5

Consequently, Financial Loss in an interaction $=$ 